\title{
Interactive comment on "Assimilating Shallow Soil Moisture Observations into Land Models with a Water Budget Constraint" by Bo Dan et al.
}

\section{Anonymous Referee \#1}

Received and published: 25 March 2020

It is with great interest that I read this paper, which investigates a number of different approaches to improve the assimilation of near-surface soil moisture observations. Specifically, the authors use an Ensemble Kalman Filter (EnKF) data assimilation (DA) framework to investigate the effect of forecast error inflation, vertical localization, and a weak water balance constraint. The find that the forecast error inflation helps to reduce the analyses error in upper soil layers that are close to the assimilated observations, however, leads to large analysis errors in deeper soil layers. They conclude that the introduction of a vertical localization function can mitigate the increased analysis error in the deeper soil layers. Finally, the authors concluded that the introduction of a weak water constraint helped to reduce the water residual after the assimilation.

Overall, this is a well-written manuscript that presents a novel and scientifically valuable 
contribution to the field of land data assimilation. I have a few minor comments that I would invite the authors to address before the publication of this manuscript.

General comments:

1. One concern I have is the authors' choice not to implement any bias correction. The argument made is that the removing the model bias can lead to issues, but what is lacking is an argument for not implementing the (arguably more traditional) approach of bias correcting the observations to match the model's climatology. By not addressing the bias between the observations and model, you are ultimately violating the assumptions of your DA framework and I would at the very least like to see a discussion on how this impacts the results.

A secondary effect of the lack of bias correction is that it probably aggravates the non-closure of the water balance. While assimilating bias-corrected observations can still lead to a water imbalance, the effect would likely be reduced and requiring less 'intervention' by the water balance constraint introduced in the 'WC' experiments. In this context, it is also worth considering that the nature of observed and modeled soil moisture can be very different (see e.g. Koster et al. 2009), which further necessitates a bias correction step.

Koster, R.D., Guo, Z., Yang, R., Dirmeyer, P.A., Mitchell, K. and Puma, M.J., 2009. On the nature of soil moisture in land surface models. Journal of Climate, 22(16), pp.4322-4335.

2. I would like to see some discussion of the feasibility of the proposed approach for global data assimilation. As the authors discuss, in particular the vertical localization function can be strongly location dependent. I am wondering whether expanding to the global domain would require computing the localization function at each model grid cell and whether that would be computationally feasible? Or would you compute a localization function at a regional scale using soil texture or climate regimes to delineate different regions? Some discussion of the transferability of the presented approach to

Printer-friendly version

Discussion paper

\section{(n)}


global scales would be valuable.

Detailed Comments:

II. 14-15: This sentence is somewhat redundant, I would recommend rephrasing it. I. 28: 'effectively reduces...' I. 42. Actually, the sub-seasonal to seasonal time scale is probably the one where the land states have the largest impact, so I would mention it here. I. 63: 'land model predictions' I. 67: 'can successfully increase' or 'successfully increases' II. 65-74: This paragraph focuses a lot on the assimilation of brightness temperatures, while your study is actually investigating methods to improve soil moisture assimilation. So I would include a discussion of soil moisture assimilation here or even a short discussion of soil moisture vs. brightness temperature assimilation. II. 115-119: Please see general comment on bias correction of observations. II.128-130: I think you are making an argument here in favor of removing the boas between model and observations. II.178-182: Just to clarify, the synthetic experiments are conducted over 40 pixels, whereas the real data are conducted only over the two ground station sites, correct? II. 230-231: I am surprised to see that you are letting the assimilated soil moisture observations update the canopy water content directly, rather than only updating the soil moisture and letting your vegetation module transport the water into the vegetation layer. Is this approach also taken for the real data assimilation experiments, when you are assimilating in situ soil moisture? I. 395: Do you mean 'At each point..'? I. 415: When you say 'the simulation case', do you mean the open loop? II. 446-449: This paragraph needs some language editing. I. 511: 'threshold'

Interactive comment on Hydrol. Earth Syst. Sci. Discuss., https://doi.org/10.5194/hess-2019696, 2020. 